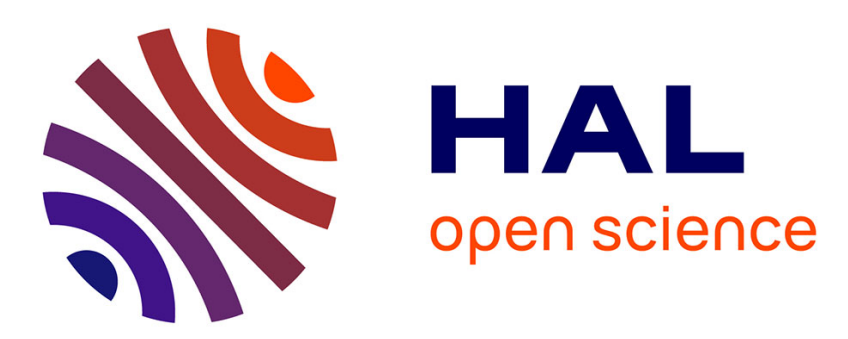

\title{
Feedback sensitivity functions analysis of finite-time stabilizing control system
}

D Zimenko, Andrey Polyakov, Denis Efimov, A Kremlev

\section{To cite this version:}

D Zimenko, Andrey Polyakov, Denis Efimov, A Kremlev. Feedback sensitivity functions analysis of finite-time stabilizing control system. International Journal of Robust and Nonlinear Control, 2016, 10.1002/rnc.3693 . hal-01405766

\section{HAL Id: hal-01405766 \\ https://hal.inria.fr/hal-01405766}

Submitted on 30 Nov 2016

HAL is a multi-disciplinary open access archive for the deposit and dissemination of scientific research documents, whether they are published or not. The documents may come from teaching and research institutions in France or abroad, or from public or private research centers.
L'archive ouverte pluridisciplinaire HAL, est destinée au dépôt et à la diffusion de documents scientifiques de niveau recherche, publiés ou non, émanant des établissements d'enseignement et de recherche français ou étrangers, des laboratoires publics ou privés. 


\title{
Feedback sensitivity functions analysis of finite-time stabilizing control system
}

\author{
K. Zimenko ${ }^{1, \dagger}$, A. Polyakov ${ }^{1,2,3}$, D. Efimov ${ }^{1,2,3}$ and A. Kremlev ${ }^{1}$ \\ ${ }^{1}$ Department of Control Systems and Informatics, ITMO University, 49 Kronverkskiy av., 197101 Saint Petersburg, \\ ${ }^{2}$ Non-A team, Inria Lille-Nord Europe, Parc Scientifique de la Haute Borne 40, avenue Halley Bat. A, Park Plaza, \\ 59650 Villeneuve d'Ascq, France \\ ${ }^{3}$ CRIStAL (UMR-CNRS 9189), Ecole Centrale de Lille, BP 48, Cite Scientifique, 59651 Villeneuve-d'Ascq, France
}

\begin{abstract}
SUMMARY
This paper presents feedback sensitivity functions analysis of implicit Lyapunov function-based control system in case of finite-time stabilization problem. The Gang of Four is chosen as a feedback sensitivity tool. The results can be used for parametric tuning of control algorithms in order to guarantee desired closed-loop sensitivity specifications. The obtained results are supported by numerical examples.
\end{abstract}

KEY WORDS: feedback sensitivity functions; Gang of Four; frequency domain design; finite-time stabilization; implicit Lyapunov function

\section{INTRODUCTION}

Modern nonlinear control theory based on state space approach to feedback design usually is not supported with frequency domain analysis, which is applied in control engineering practice. Frequently, this is the reason why control engineers use completely different tools than scientists. Due to simplicity of control parameters tuning and applicability of frequency domain analysis, PID controllers are still the most popular feedbacks in the process control today. There exists a need in development of similar tools for nonlinear control systems.

The quantitative feedback theory (QFT) is one of popular techniques in order to design a robust control in the presence of plant uncertainties and disturbances (for example, [1-7]), where desired performance is completely characterized by six feedback sensitivity transfer functions called the Gang of Six (or the Gang of Four for a system with (pure) error feedback) [1, 6, 7]. In [3, 8, 9], QFT was developed for nonlinear systems. One of ideas of these works is representation of nonlinear and multivariable systems as a parameterized family of linear time-invariant control systems and application of basic QFT approach afterwards. Nevertheless, this approach assumes to use mostly PID-based or lead-lag controllers [1-3] and other linear control techniques that are suitable for loop shaping $[6,7,10]$.

To meet the modern requirements of complex technological processes, the use of generally nonlinear control systems is necessary. For example, many controlled processes must have a finite or prescribed transient time. That is why, finite-time stability and stabilization problems have been intensively studied last years (for example, [11-15]).

\footnotetext{
${ }^{\dagger}$ E-mail: kostyazimenko@gmail.com
} 
The paper [16] is devoted to the development of a finite-time control for multiple integrators together with implicit Lyapunov function (ILF) of closed-loop system. The problem of control design for chain of integrators is quite significant because nominal models have the form of multiple integrators in some applications (for instance, different mechanical and electromechanical systems $[17,18])$. In addition, a well-developed control design technique for the chain of integrators usually admits very simple extensions to more general classes of single-input and multi-input systems, for example, feedback linearizable nonlinear control systems. An extension of results [16] for MIMO systems is presented in [19].

In order to make a proper assessment of a feedback law developed in [16] and to provide tuning rules, the present paper provides the analysis of all transfer functions in the Gang of Four. The control in [16] is essentially nonlinear and even non-Lipschitz, so frequency domain analysis of the Gang of Four and loop transfer function looks complicated. Fortunately, the control law is designed based on ILF technique [16], which allows us to interpret the nonlinear feedback as a family of linear feedbacks properly parameterized by means of the value of the Lyapunov function, and to develop a frequency domain analysis of the ILF-based control system. For processes with multiple inputs and multiple outputs [19], the obtained results may be easily extended.

Schauder principle is usually used for transformation of nonlinear systems and transition to linear control problem. However, use of this principle imposes some assumptions, for example, on a priori boundedness of all signals. For standard QFT methods with using lead-lag controllers bounds of output and input signals can be checked only posteriori [9]. The use of feedback law [16] and obtained results guarantees boundedness of the signals a priori and allows to tune control parameters for satisfying frequency domain specifications. For example, it can be applied for nonlinear systems with linear high-frequency behavior.

The paper is organized as follows. Notation used in the paper is presented in Section 2. Some preliminaries about finite-time stability, finite-time stabilization method of the multiple integrators system, and homogeneity are considered in Section 3. Problem statement is introduced in Section 4. Section 5 presents some aspects for practical implementation of the finite-time stabilization method. Frequency domain analysis of the finite-time control scheme is presented in Section 6. Section 7 presents numerical examples. Finally, conclusions with some remarks and possible directions for further works are given in Section 8.

\section{NOTATION}

Through the paper, the following notation will be used:

(i) $\mathbb{R}_{+}=\{x \in \mathbb{R}: x>0\}$, where $\mathbb{R}$ is the set of real number;

(ii) the inequality $P>0(P<0, P \geqslant 0, P \leqslant 0)$ means that $P=P^{T} \in \mathbb{R}^{n \times n}$ is symmetric and positive (negative) definite (semi-definite);

(iii) the brackets $\lfloor\cdot\rfloor$ mean rounding up to the nearest integer downwards;

(iv) diag $\left\{\lambda_{i}\right\}_{i=1}^{n}$ is the diagonal matrix with the elements $\lambda_{i}$ on the main diagonal; and

(v) a continuous function $\sigma: \mathbb{R}_{+} \cup\{0\} \rightarrow \mathbb{R}_{+} \cup\{0\}$ belongs to the class $\mathcal{K}$ if $\sigma(0)=0$ and the function is strictly increasing.

\section{PRELIMINARIES}

\subsection{Finite-time stability}

Denote a nonlinear vector field $f(t, x): \mathbb{R}_{+} \times \mathbb{R}^{n} \rightarrow \mathbb{R}^{n}$, which can be discontinuous with respect to the state variable $x \in \mathbb{R}^{n}$. Then, consider the system of the form

$$
\dot{x}=f(t, x), \quad x(0)=x_{0},
$$

the solutions $\varphi\left(t, x_{0}\right)$ of which are interpreted in the sense of Filippov [20] $f(t, 0)=0$. 
According to Filippov definition [20], an absolutely continuous function $\varphi\left(t, x_{0}\right)$ is the Cauchy problem solution associated to (1) if $\varphi\left(0, x_{0}\right)=x_{0}$ and it satisfies the following differential inclusion:

$$
\dot{x} \in K[f](t, x)=\bigcap_{\varepsilon>0} \bigcap_{\mu(N)=0} \operatorname{co} f(t, B(x, \varepsilon) \backslash N),
$$

where $\operatorname{co}(M)$ is the convex closure of the set $M, B(x, \varepsilon)$ is the ball of the radius $\varepsilon$ with the center at $x \in \mathbb{R}^{n}$, and the equality $\mu(N)=0$ means that the set $N$ has zero measure.

Assume that the origin is an equilibrium point of system (1), and it has uniqueness of solutions in forward time.

Definition 1 ([21-23])

The origin of system (1) is globally finite-time stable if

(i) finite-time attractivity: there exists a function $T: \mathbb{R}^{n} \backslash\{0\} \rightarrow \mathbb{R}_{+}$called settling time function such that $\lim _{t \rightarrow T\left(x_{0}\right)} \varphi\left(t, x_{0}\right)=0$ for any $x_{0} \in \mathbb{R}^{n} \backslash\{0\}$.

(ii) Lyapunov stability: for every $\epsilon>0$, there exists $\delta(\epsilon)>0$ such that, if $\left\|x_{0}\right\|<\delta$, then $\left\|\varphi\left(t, x_{0}\right)\right\|<\epsilon$ for every $t \geqslant 0$.

Notice that finite-time stability assumes an 'infinite eigenvalue assignment' for the system at the origin.

\subsection{Homogeneity}

Homogeneity [24-26] is an intrinsic property of such objects as functions or vector fields, which remains consistent with respect to some scaling operation called a dilation.

For fixed $r_{i} \in \mathbb{R}_{+}, i=\overline{1, n}$ and $\lambda>0$, one can define the vector of weights $r=\left(r_{1}, \ldots, r_{n}\right)^{T}$ and the dilation matrix $D(\lambda)=\operatorname{diag}\left\{\lambda^{r_{i}}\right\}_{i=1}^{n}$. Note that $D(\lambda) x=\left(\lambda^{r_{1}} x_{1}, \ldots, \lambda^{r_{n}} x_{n}\right)^{T}$ represents a mapping $x \mapsto D(\lambda) x$ called a dilation for $x \in \mathbb{R}^{n}$.

Definition 2 ([26])

A function $g: \mathbb{R}^{n} \rightarrow \mathbb{R}$ (vector field $f: \mathbb{R}^{n} \rightarrow \mathbb{R}^{n}$ ) is said to be $r$-homogeneous of degree $m$ iff $g(D(\lambda) x)=\lambda^{m} g(x)\left(f(D(\lambda) x)=\lambda^{m} D(\lambda) f(x)\right)$ for all $\lambda>0$ and $x \in \mathbb{R}^{n}$.

Theorem 1 ([27])

Let $f$ be a $r$-homogeneous continuous vector field on $\mathbb{R}^{n}$ with a negative degree. Then, if the system $\dot{x}=f(x)$ is a locally asymptotically stable, it is globally finite-time stable.

In addition, the homogeneity theory provides many advantages to analysis and design of nonlinear control system (for example, $[28,29]$ ).

\subsection{Finite-time stabilization for multiple integrators}

Consider a single-input control system of the following form:

$$
\dot{x}=A x+b u+d(t, x),
$$

where $x \in \mathbb{R}^{n}$ is the state vector, $u \in \mathbb{R}$ is the control input,

$$
A=\left(\begin{array}{ccccc}
0 & 1 & 0 & \cdots & 0 \\
0 & 0 & 1 & \cdots & 0 \\
\vdots & \vdots & \vdots & \ddots & \vdots \\
0 & 0 & 0 & \cdots & 1 \\
0 & 0 & 0 & \cdots & 0
\end{array}\right) \quad \text { and } \quad b=\left(\begin{array}{c}
0 \\
0 \\
\vdots \\
0 \\
1
\end{array}\right)
$$

and the function $d(t, x): \mathbb{R}^{n+1} \rightarrow \mathbb{R}^{n}$ describes the system uncertainties and disturbances. Note that for $d(t, x) \equiv 0$, system (2) describes a chain of integrators. 
Introduce the function

$$
Q(V, x):=x^{T} D\left(V^{-1}\right) P D\left(V^{-1}\right) x-1,
$$

where $P=P^{T} \in \mathbb{R}^{n \times n}$ is a symmetric positive definite matrix and $D(\lambda)$ is the dilation matrix of the form

$$
D(\lambda)=\operatorname{diag}\left\{\lambda^{1+(n-i) \mu}\right\}_{i=1}^{n}, \quad 0<\mu \leqslant 1 .
$$

Denote the matrix $H_{\mu}=\operatorname{diag}\{1+(n-i) \mu\}_{i=1}^{n}$.

Theorem $2([16,19])$

If

(i) the system of matrix inequalities:

$$
\left\{\begin{array}{l}
A X+X A^{T}+b y+y^{T} b^{T}+H_{\mu} X+X H_{\mu}+\beta I_{n} \leqslant 0, \\
X H_{\mu}+H_{\mu} X>0, \quad X>0,
\end{array}\right.
$$

is feasible for some $\mu \in(0,1], \beta \in(0,1), X \in \mathbb{R}^{n \times n}$ and $y \in \mathbb{R}^{1 \times n}$;

(ii) the control $u(V, x)$ has the form

$$
u(V, x)=V^{1-\mu} k D\left(V^{-1}\right) x,
$$

where $k=\left(k_{1}, \ldots, k_{n}\right)=y X^{-1}$,

$$
V \in \mathbb{R}_{+}: Q(V, x)=0
$$

and $Q(V, x)$ is presented by (3) with $P=X^{-1}$;

(iii) the disturbance function $d(t, x)$ satisfies the following inequality:

$$
d^{T}(t, x) D^{2}\left(V^{-1}\right) d(t, x) \leqslant \beta^{2} V^{-2 \mu} x^{T} D\left(V^{-1}\right)\left(H_{\mu} P+P H_{\mu}\right) D\left(V^{-1}\right) x ;
$$

Then, system (2) is globally finite-time stable, and the settling time function estimate has the form

$$
T\left(x_{0}\right) \leqslant \frac{V_{0}^{\mu}}{\mu(1-\beta)},
$$

where $V_{0} \in \mathbb{R}_{+}: Q\left(V_{0}, x_{0}\right)=0$.

In disturbance-free case $\beta$ tends to zero, inequality (6) gives $d(t, x) \equiv 0$, and the conditions of Theorem 2 coincide with Theorem 3 in [16].

Inequality (6) presents restriction to disturbance function $d(t, x)$ and has an implicit form, which may be not appropriate for practice. To expose the restrictions to the function $d(t, x)$, the following proposition can be applied.

Proposition 1 ([19])

Let $X \in \mathbb{R}^{n \times n}$ be a solution of LMI system (4) and $d=\left(d_{1}, d_{2}, \ldots, d_{n}\right)^{T}$. If

$$
d_{i}^{2} \leqslant \beta \beta_{i} \chi\left\{\begin{array}{lll}
\left(\lambda_{\min }(P) x^{T} x\right)^{1+(n-i-1) \mu} & \text { if } \quad x^{T} P x \leqslant 1, \\
\left(\lambda_{\min }(P) x^{T} x\right)^{\frac{1+(n-i-1) \mu}{1+(n-1) \mu}} & \text { if } \quad x^{T} P x>1,
\end{array}\right.
$$

for some $\beta_{i} \in \mathbb{R}_{+}: \beta=\beta_{1}+\cdots+\beta_{n}<1$ and $\left.\chi:=\lambda_{\min }\left(P^{1 / 2} H_{\mu}\right) P^{-1 / 2}+P^{-1 / 2} H_{\mu}\right) P^{1 / 2}$, then inequality (6) of Theorem 2 holds.

Restriction (6) implies that the disturbance $d(t, x)$ may not vanish at the origin (for example, $d_{n}(t, x)$, can be simply bounded if $\left.\mu=1\right)$, and may be non-Lipschitz or discontinuous like a dry friction. This issue makes presented control law attractive for a number of control applications, for example, friction electromechanical system [30] and cart-pendulum system [31]. 


\section{PROBLEM STATEMENT}

A block diagram of a basic feedback scheme for systems where feedback is restricted to operate on the error signal is shown in Figure 1, where $P$ is the process to be controlled; $C$ is the control block; $u$ is the control signal; $\eta$ is the process output; $y$ is the plant output; and the external signals are denoted by the reference signal $r$, the measurement noise $n$, and the load disturbance $d$.

In this case, the system in Figure 1 is completely characterized by four transfer functions called the Gang of Four: the sensitivity function $S$, the complementary sensitivity function $T$, the load disturbance sensitivity function $P S$, and the noise sensitivity function $C S$. The closed-loop specifications are typically defined in terms of inequalities on amplitude frequency responses of the Gang of Four transfer functions (moreover, the main specifications in practice are either based on the Gang of Four, or can be translated to it [4]):

(i) Noise attenuation at the plant output

$$
|S(j \omega)|=\left|\frac{1}{1+P(j \omega) C(j \omega)}\right| \leqslant \beta_{S}(\omega), \quad \forall \omega>0 .
$$

The sensitivity function $S$ gives the response of the plant output to the noise measurement and describes how noises are attenuated by closing the feedback loop (Noises are attenuated if $|S(j \omega)|<1$ and amplified if $|S(j \omega)|>1$ and the maximum sensitivity $M_{s}=\max _{\omega}|S(j \omega)|$ on some frequency $\omega_{m s}$ corresponds to the largest amplification of the noises; the frequency where $|S(j \omega)|=1$ is called the sensitivity crossover frequency $\omega_{s c}$.)

(ii) Stability

$$
|T(j \omega)|=\left|\frac{P(j \omega) C(j \omega)}{1+P(j \omega) C(j \omega)}\right|<\lambda_{T}, \quad \forall \omega>0 .
$$

The complementary sensitivity function $T$ gives the response of the control variable to the load disturbance. Inequality (9) implies that the closed-loop system is stable for substantial variations in the process dynamics, namely, variations can be large for those frequencies $\omega$ where $|T(j \omega)|$ is small and smaller variations are permitted for frequencies $\omega$ where the value $|T(j \omega)|$ is large.

(iii) Disturbance rejection

$$
|P S(j \omega)|=\left|\frac{P(j \omega)}{1+P(j \omega) C(j \omega)}\right| \leqslant \beta_{P S}(\omega), \quad \forall \omega>0 .
$$

The load disturbance sensitivity function $P S$ gives the response of the process output to the load disturbance. Because load disturbances typically have low frequencies, $|P S(j \omega)|$ typically has smaller value at low frequencies.

(iv) Noise rejection

$$
|C S(j \omega)|=\left|\frac{C(j \omega)}{1+P(j \omega) C(j \omega)}\right| \leqslant \beta_{C S}(\omega), \quad \forall \omega>0 .
$$

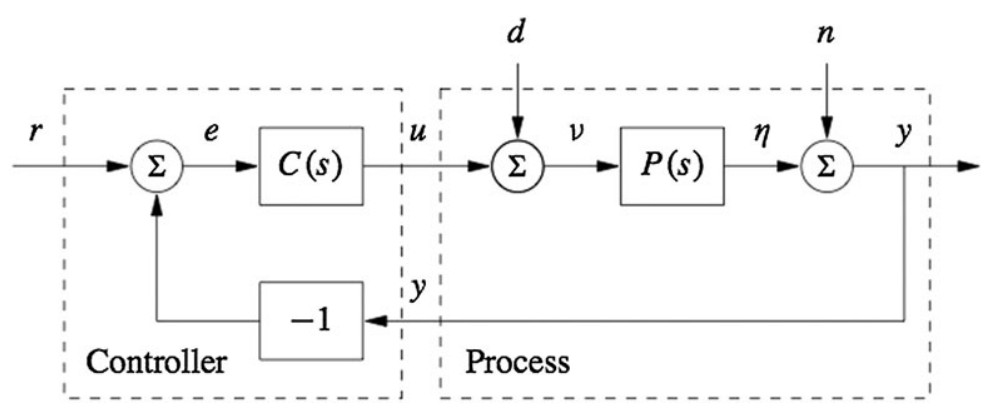

Figure 1. Block diagram of a linear feedback loop. 
The noise sensitivity function $C S$ gives the response of the control variable to the noise. Because noise signals typically have high frequencies, $|C S(j \omega)|$ typically has smaller value at high frequencies, and the smaller the value $|C S(j \omega)|$ the less the effect of noise on the process.

The main aim of this paper is to adapt the analysis of the Gang of Four to the nonlinear feedback systems (2) and (5), and to provide tuning rules in order to meet the desired closed-loop specifications.

\section{PRACTICAL IMPLEMENTATION OF THE ILF CONTROL}

The implementation of the control scheme presented in Theorem 2 requires to solve the equation $Q(V, x)=0$ in order to find the ILF value $V$. In some cases, the function $V(x)$ can be calculated analytically (for instance, the paper [16] contains an example of analytical calculation of $V$ for $n=2$ ). However, generally, these calculations are very cumbersome.

In this case, the discrete-time version of the control scheme can be implemented with using a simple numerical procedure in order to find a corresponding value of $V_{i}$ at the time instant $t_{i}$ $[16,19]$.

Denote an arbitrary sequence of time instances $\left\{t_{i}\right\}_{i=0}^{+\infty}$, where $0=t_{0}<t_{1}<t_{2}<\ldots$.

Corollary 1 ([19])

Let the conditions of Theorem 2 hold, then the origin of the system (2) is asymptotically stable with the switching control

$$
u(x)=u\left(V_{i}, x\right) \quad \text { for } \quad t \in\left[t_{i}, t_{i+1}\right),
$$

where $V_{i}>0: Q\left(V_{i}, x\left(t_{i}\right)\right)=0$.

The Corollary 1 shows that the sampled-time control in the form (5) keeps the robust stability property of the closed-loop system (2) independently on the sampling interval. The control (12) is piecewise linear, and for any fixed $V$ between two switching instants, the system (2) becomes a linear, where $V \in\left[V_{\min }, V_{0}\right], V_{0} \in \mathbb{R}_{+}: Q\left(V_{0}, x_{0}\right)=0$.

Note that this result allows to consider the specifications that are time-dependent, for example, $\left|S\left(V\left(t_{i}\right), j \omega\left(t_{i}\right)\right)\right| \leqslant \beta_{S}\left(\omega\left(t_{i}\right)\right)$.

The parameter $V_{\min }$ can be selected by control engineers using the frequency domain analysis to be developed below, with considering $V$ as a scalar parameter of the linear control. The parameter $V_{\min }$ defines lower admissible value of $V$ and cannot be selected arbitrary small in practice because of finite numerical precision of digital devices and measurement errors.

\section{FREQUENCY DOMAIN ANALYSIS}

\subsection{The Gang of Four derivation}

From (2) and (5), the transfer functions in the blocks according to Figure 1 at each time instance take the following form:

$$
P=\frac{1}{S^{n}}
$$

and

$$
C=-s^{n-1} V^{-\mu} k_{n}-s^{n-2} V^{-2 \mu} k_{n-1}-\cdots-V^{-n \mu} k_{1} .
$$

Amplitude frequency responses for the Gang of Four transfer functions (8)-(11) take the forms:

$$
|S(j \omega)|=\frac{1}{\sqrt{(1-a)^{2}+b^{2}}},
$$




$$
\begin{gathered}
|T(j \omega)|=\frac{\sqrt{c^{2}+d^{2}}}{\omega^{n} \sqrt{(1-a)^{2}+b^{2}}}, \\
|P S(j \omega)|=\frac{1}{\omega^{n} \sqrt{(1-a)^{2}+b^{2}}}, \\
|C S(j \omega)|=\frac{\sqrt{c^{2}+d^{2}}}{\sqrt{(1-a)^{2}+b^{2}}},
\end{gathered}
$$

where

$$
\begin{gathered}
a=\sum_{i=1}^{\left\lfloor\frac{n}{2}\right\rfloor} \frac{(-1)^{i} k_{n+1-2 i}}{\omega^{2 i} V^{2 i \mu}}, \quad b=\sum_{i=0}^{\left\lfloor\frac{n+1}{2}\right\rfloor-1} \frac{(-1)^{i+1} k_{n-2 i}}{\omega^{2 i+1} V^{(2 i+1) \mu}}, \\
c=\sum_{i=0}^{\left\lfloor\frac{n+1}{2}\right\rfloor-1} \frac{(-1)^{i} \omega^{2 i} k_{2 i+1}}{V^{(n-2 i) \mu}}, \quad d=\sum_{i=1}^{\left\lfloor\frac{n}{2}\right\rfloor} \frac{(-1)^{i-1} \omega^{2 i-1} k_{2 i}}{V^{(n-2 i+1) \mu}} .
\end{gathered}
$$

\subsection{The Gang of Four analysis}

Because $V$ parameterizes the control, let us consider the limits of the Gang of Four (13)-(16) for $\omega \rightarrow+\infty$ and $\omega \rightarrow 0$ when $V=$ const $:$

$$
\begin{gathered}
\lim _{\omega \rightarrow+\infty}|S(j \omega)|=1, \quad \lim _{\omega \rightarrow+\infty}|T(j \omega)|=0, \\
\lim _{\omega \rightarrow+\infty}|P S(j \omega)|=0, \quad \lim _{\omega \rightarrow+\infty}|C S(j \omega)|=\infty, \\
\lim _{\omega \rightarrow 0}|S(j \omega)|=0, \quad \lim _{\omega \rightarrow 0}|T(j \omega)|=1, \\
\lim _{\omega \rightarrow 0}|P S(j \omega)|=\frac{V^{-n \mu}}{\left|k_{1}\right|}, \quad \lim _{\omega \rightarrow 0}|C S(j \omega)|=0,
\end{gathered}
$$

and limits for $V \rightarrow 0$ when $\omega=$ const:

$$
\begin{array}{cc}
\lim _{V \rightarrow 0}|S(j \omega)|=0, & \lim _{V \rightarrow 0}|T(j \omega)|=1, \\
\lim _{V \rightarrow 0}|P S(j \omega)|=0, & \lim _{V \rightarrow 0}|C S(j \omega)|=\omega^{n} .
\end{array}
$$

Looking at limits for $C S$, one can conclude that the system reduces the influence of noise only at significantly low frequencies; then, there is a little consolation as noise usually tends to be present at high frequencies. However, it is well known that good reference signal tracking and disturbance rejection has to be traded off against suppression of process noise.

The sensitivity function $S$ is close to 1 for high frequencies and $C S$ can be approximated as

$$
C S=C * S \approx C .
$$

Thus, because measurement noise typically has high frequencies, the high-frequency gain of the controller is thus an important characteristic. To reduce the high-frequency gain of the controller $C$ and thereby reduce the value $|C S(j \omega)|$, the case of decreasing $\left|k_{i}\right|, i=\overline{1, n}$ or/and increasing the parameter $V_{\min }$ can be considered. However, the selection of too large value of the parameter $V_{\min }$ is also undesirable because the ILF control provides the stabilization of the system with the attractive set $\Pi_{\mu}\left(V_{\min }, P\right):=\left\{z \in \mathbb{R}^{n}: z^{T} D\left(V_{\min }^{-1}\right) P D\left(V_{\min }^{-1}\right) z \leqslant 1\right\}[19]$. 


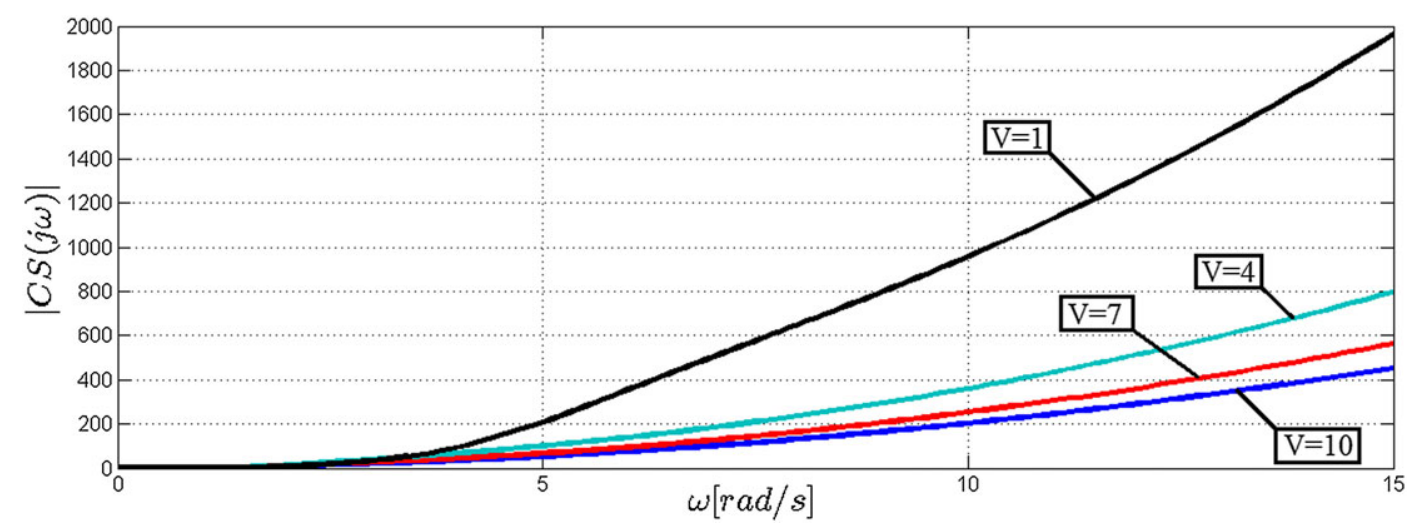

Figure 2. Gain curve of the noise sensitivity function $C S$.

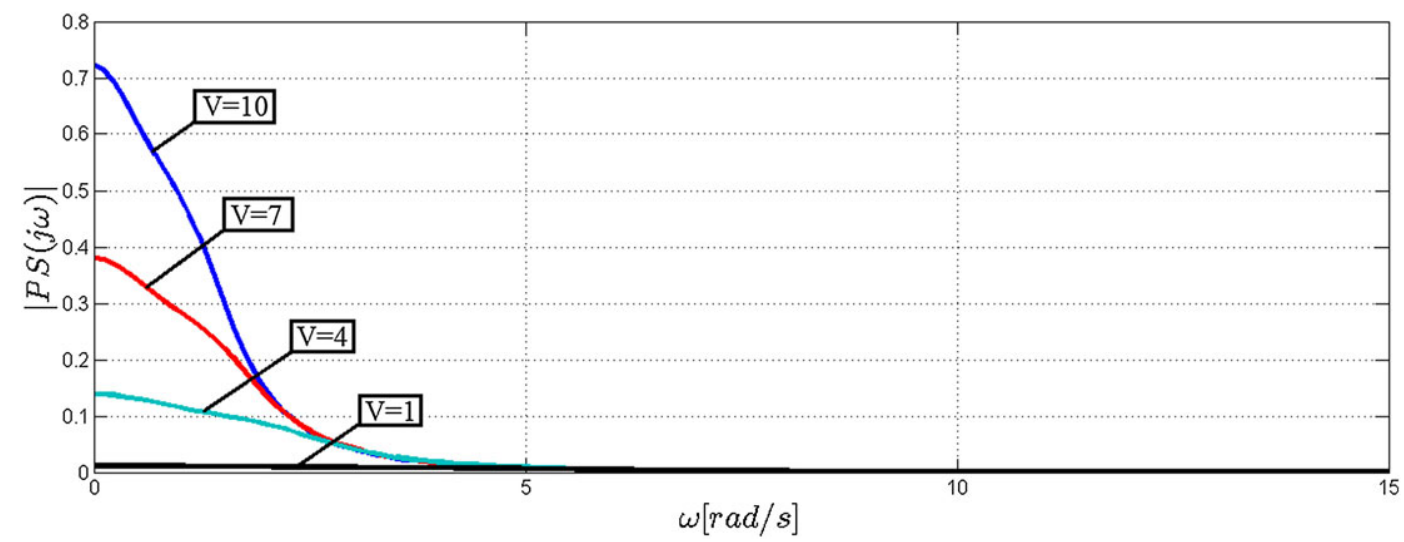

Figure 3. Gain curve of the load disturbance sensitivity function $P S$.

The gain curve of the noise sensitivity function for $n=3, k=(-87.4154-46.7109-7.9769)$, $\mu=0.6$ and different values of $V$ is shown in Figure 2.

Let us consider the load disturbance sensitivity function $P S$. Because load disturbances typically have low frequencies, it is natural to focus on the behavior of the transfer function at low frequencies. As $\lim _{\omega \rightarrow 0}|T(j \omega)|=1$, we have the following approximation for small $\omega$ :

$$
P S=\frac{T}{C} \approx \frac{1}{C} .
$$

Thus, the greater $\left|k_{i}\right|, i=\overline{1, n}$ or/and smaller $V$, the smaller the value of $P S$. Finally, as $\lim _{V \rightarrow 0}|P S(j \omega)|=0$, the influence of the load disturbance disappears completely. The gain curve of the load disturbance sensitivity function for the same parameters is shown in Figure 3.

Based on continuity of the Gang of Four functions in $\mathbb{R}^{n} \backslash\{0\}$ the next proposition has been achieved.

\section{Proposition 1}

For any $\beta_{P S}>0\left(\beta_{S}>0\right)$ and $\omega_{P S}>0\left(\omega_{S}>0\right)$, there is $V_{P S}>0\left(V_{S}>0\right)$ such that $|P S(\omega)| \leqslant$ $\beta_{P S}\left(|S(j \omega)| \leqslant \beta_{S}\right)$ for all $\omega \leqslant \omega_{P S}\left(\omega \leqslant \omega_{S}\right)$ and $V \leqslant V_{P S}\left(V \leqslant V_{S}\right)$.

Also, one can conclude that amplitude frequency responses of the Gang of Four functions are homogeneous:

\section{Proposition 2}

Amplitude frequency responses of the sensitivity $\left|S\left(\omega, V^{-1}\right)\right|$ and complementary sensitivity $\left|T\left(\omega, V^{-1}\right)\right|$ functions are homogeneous of zero degree with the vector of weights $r=\left(1, \mu^{-1}\right)^{T}$. 
Amplitude frequency responses of the load disturbance sensitivity $\left|P S\left(\omega, V^{-1}\right)\right|$ and noise sensitivity $\left|C S\left(\omega, V^{-1}\right)\right|$ functions are homogeneous of degrees $-n$ and $n$, respectively, with the same vector of weights $r=\left(1, \mu^{-1}\right)^{T}$.

Proof

Let us look at $a$ and $b$ as functions of two variables $\omega$ and $\tau=V^{-1}$. Then,

$$
\begin{gathered}
a\left(\lambda \omega, \lambda^{\frac{1}{\mu}} \tau\right)=\sum_{i=1}^{\left\lfloor\frac{n}{2}\right\rfloor} \frac{(-1)^{i} k_{n+1-2 i}\left(\lambda^{\frac{1}{\mu}} \tau\right)^{2 i \mu}}{(\lambda \omega)^{2 i}}=a(\omega, \tau), \\
b\left(\lambda \omega, \lambda^{\frac{1}{\mu}} \tau\right)=\sum_{i=0}^{\left\lfloor\frac{n+1}{2}\right\rfloor-1} \frac{(-1)^{i+1} k_{n-2 i}\left(\lambda^{\frac{1}{\mu}} \tau\right)^{(2 i+1) \mu}}{(\lambda \omega)^{2 i+1}}=b(\omega, \tau)
\end{gathered}
$$

and thus,

$$
|S(\omega, \tau)|=\left|S\left(\lambda \omega, \lambda^{\frac{1}{\mu}} \tau\right)\right|,
$$

that is, the function $\left|S\left(\omega, V^{-1}\right)\right|$ is homogeneous of zero degree with the vector of weights $r=$ $\left(1, \mu^{-1}\right)^{T}$.

Proofs for other Gang of Four functions are similar and based on the following expressions:

$$
c\left(\lambda \omega, \lambda^{\frac{1}{\mu}} \tau\right)=\lambda^{n} c(\omega, \tau)
$$

and

$$
d\left(\lambda \omega, \lambda^{\frac{1}{\mu}} \tau\right)=\lambda^{n} d(\omega, \tau)
$$

Based on Proposition 2, one can conclude that at each mode $V=V_{i}$, the sensitivity functions can be obtained through the previous one. For example, the sensitivity function $S\left(\omega, V_{i}\right)$ can be represented as

$$
\left|S\left(\omega, V_{i}\right)\right|=\left|S\left(\left(\frac{V_{i}}{V_{i-1}}\right)^{\mu} \omega, V_{i-1}\right)\right| .
$$

Thus, the sensitivity crossover frequency $\omega_{s c}$ and the maximum value frequency $\omega_{m s}$ can be also obtained through previous values, and the maximum sensitivity $M_{S}$ is constant independently on the ILF value $V$. The gain curve of the sensitivity function is shown in Figure 4.

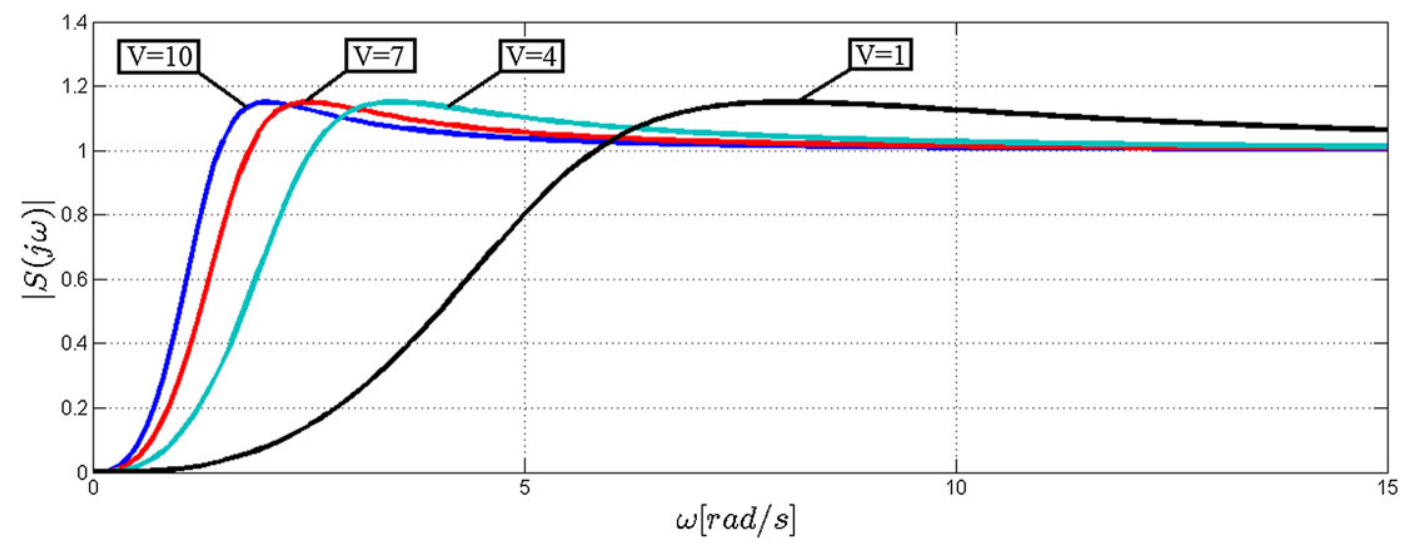

Figure 4. Gain curve of the sensitivity function $S$. 


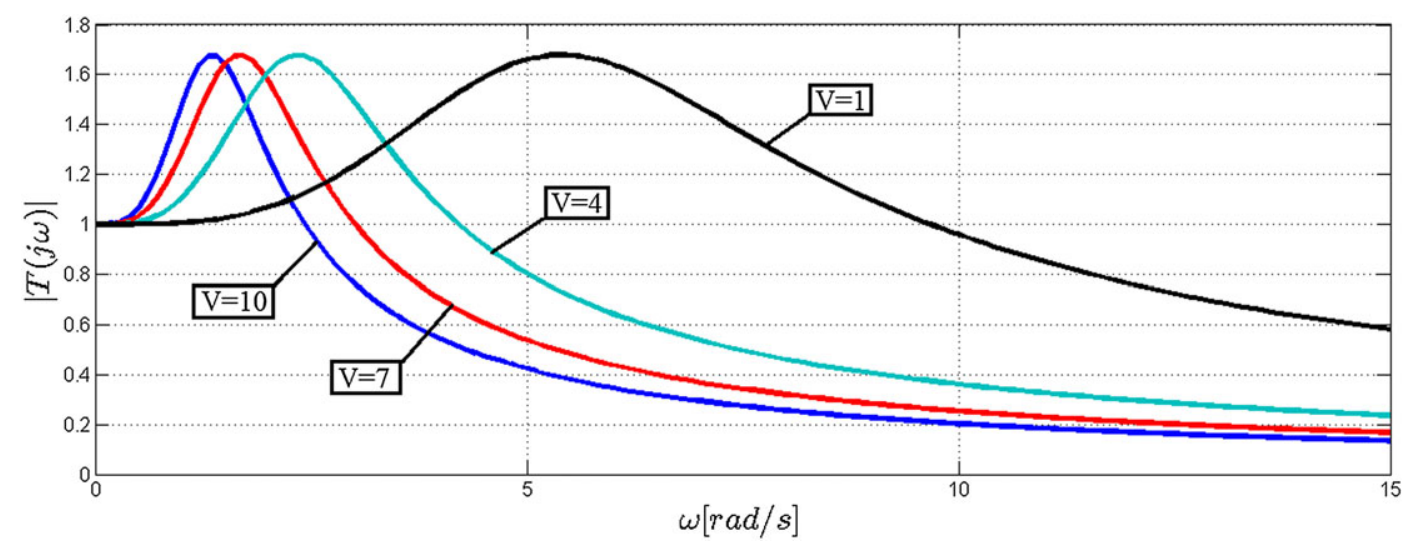

Figure 5. Gain curve of the complementary sensitivity function $T$.

The similar conclusions can be made about the complementary sensitivity function $T$ (Figure 5).

\section{Remark 1}

Because $\left\{x: V(x)<V_{\min }\right\}$ is the main operation domain of the system after the initial (finite-time) transition, one can select well-tuned linear controller in the form $u=\tilde{k} x, \tilde{k}=\left(\tilde{k}_{1}, \tilde{k}_{2}, \ldots, \tilde{k}_{n}\right)$ and design an ILF control (5) for

$$
k=V_{\min }^{\mu-1} \tilde{k} D\left(V_{\min }\right)
$$

by means of homogeneous scaling. This transformation allows to keep all frequency domain characteristics for $\left\{x: V(x)<V_{\min }\right\}$.

Because there are many processes that can be described by the second-order plants (for instance, mechanical planar systems), more detailed results are presented in the succeeding discussion for $n=2$.

\subsection{Second-order plant}

According to [19], the first matrix inequality of the system (4) for $d(t, x) \equiv 0, \beta=0$ can be replaced with the equality

$$
\left\{\begin{array}{l}
x_{i+1}+[1+\mu(n-i)] x_{i i}=0, \\
x_{i+1 j}+x_{i j+1}+[2+\mu(2 n-i-j)] x_{i j}=0, \\
x_{i+1 n}+[2+\mu(n-i)] x_{i n}+y_{i}=0 \\
x_{n}+y_{n}=0
\end{array}\right.
$$

where $j>i=\overline{1, n-1}$, and according to (21) for $n=2$, the matrices $X$ and $y$ take the forms

$$
\begin{gathered}
X=\left(\begin{array}{cc}
x_{11} & -(1+\mu) x_{11} \\
-(1+\mu) x_{11} & x_{22}
\end{array}\right), \\
y=\left((2+\mu)(1+\mu) x_{11}-x_{22}-x_{22}\right),
\end{gathered}
$$

where $x_{11}$ and $x_{22}$ are chosen in order to $X>0$ and $X H_{\mu}+H_{\mu} X>0$, that is, $x_{11}>0$ and $x_{22}>x_{11}(1+\mu)^{2}$.

As $k=\left(k_{1}, k_{2}\right)=y X^{-1}$, one can obtain $k_{1}=-\frac{x_{22}}{x_{11}}$ and $k_{2}=-2-\mu$. Thus, for $n=2$, the coefficients $k_{1}$ and $k_{2}$ can take the following values:

$$
k_{1}<-0.25(1+\mu)(2+\mu)^{2}
$$

and

$$
k_{2}=-2-\mu .
$$




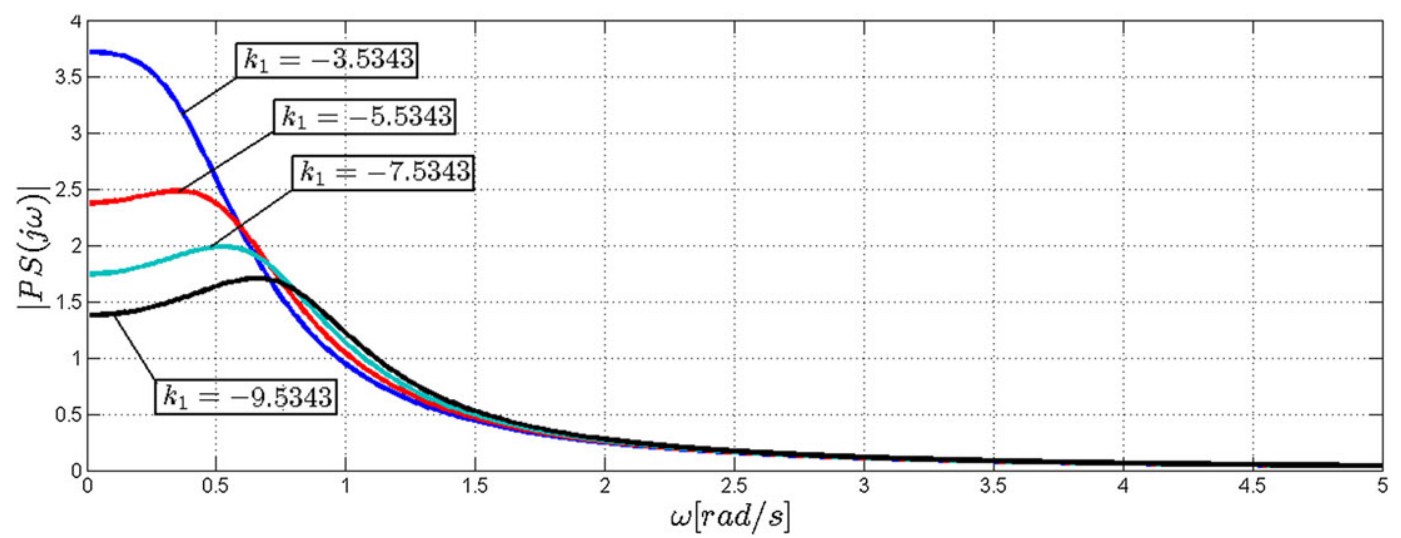

Figure 6. Gain curve of the load disturbance sensitivity function $P S$ for the second-order plant.

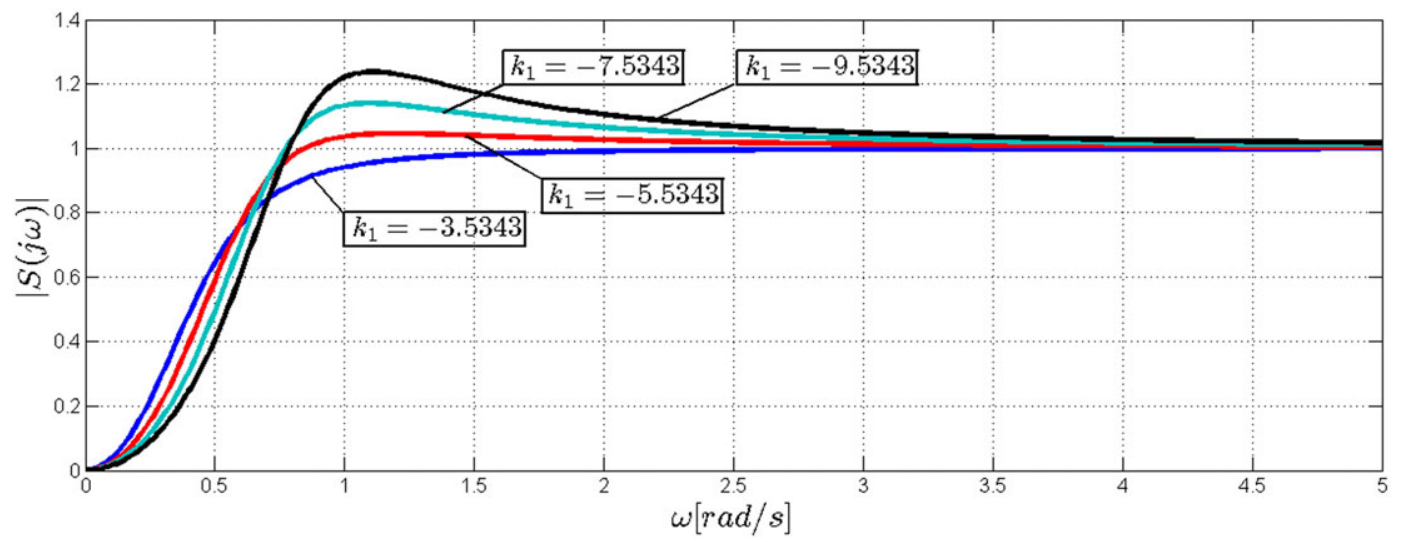

Figure 7. Gain curve of the sensitivity function $S$ for the second-order plant.

Thus, according to (18), decreasing $k_{1}$ allows to get better attenuation of load disturbances at low frequencies (Figure 6, where $\mu=0.8, V=5$ ).

However, as for $n=2$, the amplitude frequency response for sensitivity function according to (13) takes the form

$$
|S(j \omega)|=\frac{\omega^{2}}{\sqrt{\omega^{4}+\left(2 k_{1}+k_{2}^{2}\right) V^{-2 \mu} \omega^{2}+k_{1}^{2} V^{-4 \mu}}},
$$

one can conclude that selection of $k_{1} \in\left(-0.5(2+\mu)^{2},-0.25(1+\mu)(2+\mu)^{2}\right)$ implies noises attenuation $|S(j \omega)|<1$ for all finite frequencies (for example, Figure 7). For $\left|k_{1}\right|>0.5(2+\mu)^{2}$, the function $|S(j \omega)|$ has maximum $M_{s}=\frac{-2 k_{1}}{\sqrt{-4 k_{1} k_{2}^{2}-k_{2}^{4}}}$ for any value $V$ at frequency $\omega_{m s}=$ $\sqrt{\frac{-2 k_{1}^{2} V^{-2 \mu}}{2 k_{1}+k_{2}^{2}}}$. Thus, the greater $\left|k_{1}\right|>0.5(2+\mu)^{2}$, the greater value $M_{s}$ at greater frequency $\omega_{m s}$.

The amplitude frequency response for complementary sensitivity function $T$ takes the form

$$
|T(j \omega)|=\frac{\sqrt{\omega^{2} V^{-2 \mu} k_{2}^{2}+V^{-4 \mu} k_{1}^{2}}}{\sqrt{\omega^{4}+\left(2 k_{1}+k_{2}^{2}\right) V^{-2 \mu} \omega^{2}+k_{1}^{2} V^{-4 \mu}}}
$$


and

$$
|T(j \omega)|_{\max }=\sqrt{\frac{k_{2}^{4} \sqrt{k_{1}^{2}-2 k_{2}^{2} k_{1}}}{-2 k_{1}^{3}+4 k_{2}^{2} k_{1}^{2}+\sqrt{k_{1}^{2}-2 k_{2}^{2} k_{1}\left(-2 k_{1}^{2}+2 k_{1} k_{2}^{2}+k_{2}^{4}\right)}}}
$$

for any value $V$ at frequency $\omega=-k_{2}^{-1} V^{-\mu} \sqrt{-k_{1}^{2}+\sqrt{k_{1}^{4}-2 k_{2}^{2} k_{1}^{3}}}$. Therefore, as $|T(j \omega)|_{\max }$ does not depend on the values of $\omega$ and $V$, we can rewrite specification (9)

$$
\sqrt{\frac{k_{2}^{4} \sqrt{k_{1}^{2}-2 k_{2}^{2} k_{1}}}{-2 k_{1}^{3}+4 k_{2}^{2} k_{1}^{2}+\sqrt{k_{1}^{2}-2 k_{2}^{2} k_{1}}\left(-2 k_{1}^{2}+2 k_{1} k_{2}^{2}+k_{2}^{4}\right)}} \leqslant \lambda_{T}
$$

and the greater $\left|k_{1}\right|>0.25(1+\mu)(2+\mu)^{2}$, the greater value $|T(j \omega)|_{\max }$ at greater frequency $\omega_{m t}$. Thereby, to fulfill the specification (9), the selection of the parameter $k_{1}<-0.25(1+\mu)(2+\mu)^{2}$ close to its maximum value is preferable.

The gain curve of the complementary sensitivity function $T$ for the same values as in Figures 6 and 7 is shown in Figure 8.

Because $C S=\omega^{2} T$ according to (14) and (16), the selection of the parameter $k_{1}$ close to its maximum value is preferable to fulfill the specification (11). The gain curve of the noise sensitivity function $C S$ is shown in Figure 9 for different values of $k_{1}$.

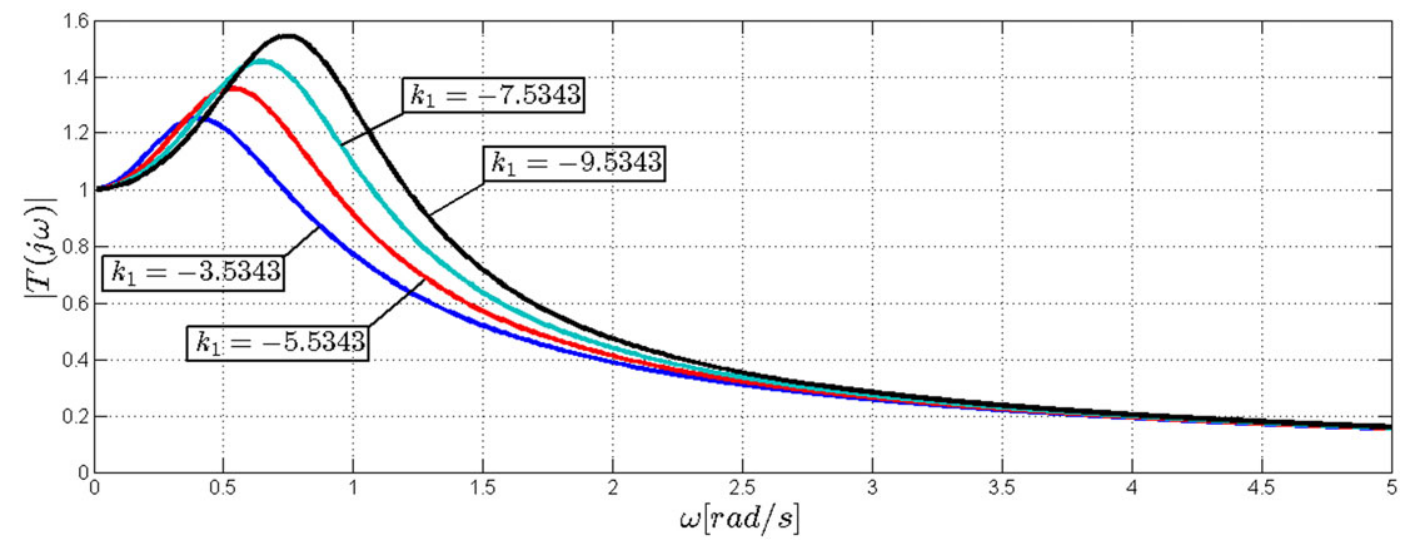

Figure 8. Gain curve of the complementary sensitivity function $T$ for the second-order plant.

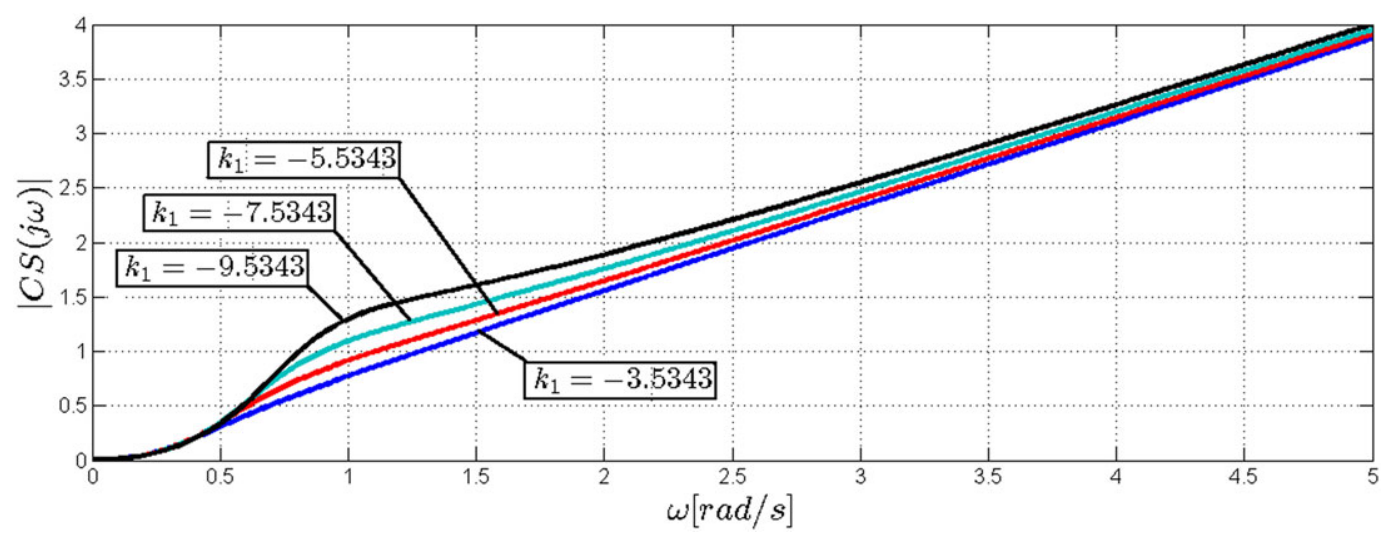

Figure 9. Gain curve of the noise sensitivity function $C S$ for the second-order plant. 


\section{NUMERICAL EXAMPLES}

\subsection{Roll control for a vectored thrust aircraft}

The loop transfer function $L=P C=\frac{T}{S}$ also can be considered for frequency domain design using the same finite-time control scheme. As an example, let us consider the problem of roll control for a vectored thrust aircraft presented in [1]. The simplified model represents the double integrator system with some gain in the form

$$
P(s)=\frac{r}{J s^{2}},
$$

where $r=0.25 \mathrm{~m}$ is the force moment arm and $J=0.0475 \mathrm{~kg} \mathrm{~m}^{-2}$ is the vehicle inertia.

Assume that the system has to meet the following performance specifications:

(i) the error in steady state is less than $1 \%$; and

(ii) the tracking error is less than $10 \%$ up to $10 \mathrm{rad} \mathrm{s}^{-1}$.

To achieve this performance specifications, it is necessary to increase the crossover frequency $\omega_{s c}$ in order to have a gain at least 10 at a frequency of $10 \mathrm{rad} \mathrm{s}^{-1}$, where $\omega_{s c}=\left\{\omega \in \mathbb{R}_{+}:|L(j \omega)|=1\right\}$ for the loop transfer function $L$. Absolute value of the loop transfer function $L$ takes the form

$$
|L(j \omega)|=\frac{r}{J} \sqrt{\omega^{-4} V^{-4 \mu} k_{1}^{2}+\omega^{-2} V^{-2 \mu} k_{2}^{2}} .
$$

It is easy to see that the value of the crossover frequency is increased for $V$ tending to zero (Figure 10) and for greater values of $\left|k_{1}\right|$ (Figure 11). However, in the consideration of an argument of the loop transfer function

$$
\angle L(j \omega)=\arctan \left(\frac{\omega V^{\mu} k_{2}}{k_{1}}\right)
$$

one can conclude that rather large values of $\left|k_{1}\right|$ give a very low-phase margin (Figure 11) and as the functions $|L(j \omega, V)|$ and $\angle L(j \omega, V)$ are homogeneous of zero degree then phase margin is constant independently on the ILF value $V$ (Figure 10).

Thus, to satisfy the performance specifications and provide high-phase margin, the coefficient $k_{1}$ should be chosen close to the value $-0.25(1+\mu)(2+\mu)^{2}$.

\subsection{Nonlinear plants with linear high-frequency behavior}

Consider an example of a plant with input nonlinearity:

$$
\frac{d^{2} y(t)}{d t^{2}}=u(t)+c u^{3}(t)
$$

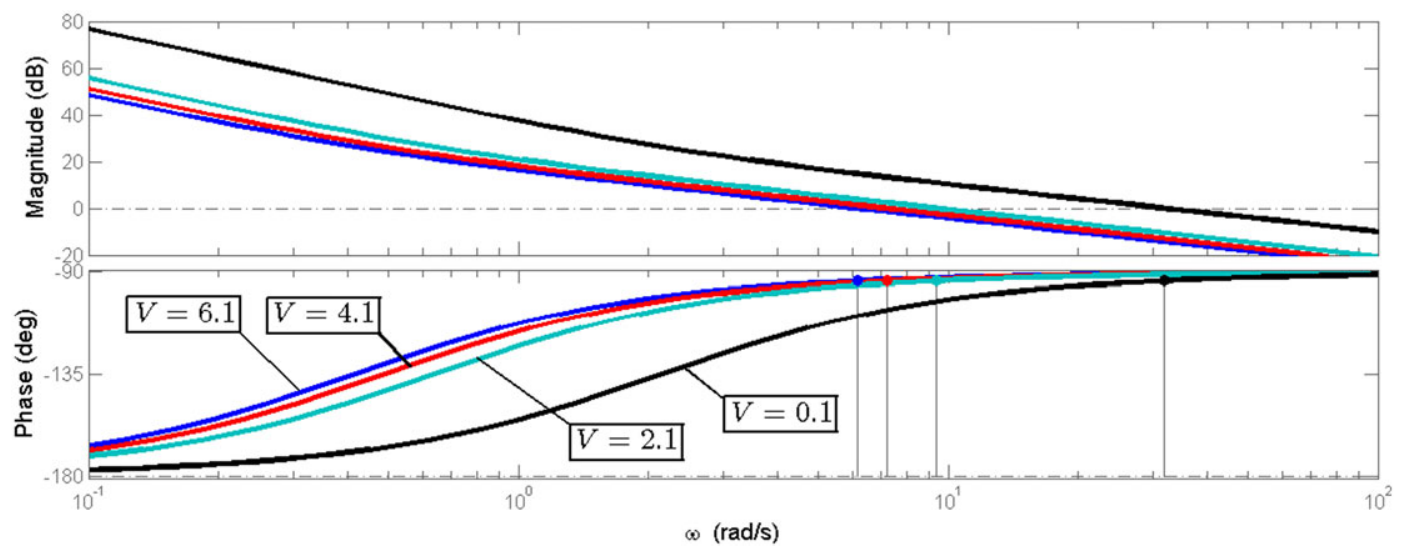

Figure 10. Bode plot for the loop transfer function $L$ with different values of $V$. 


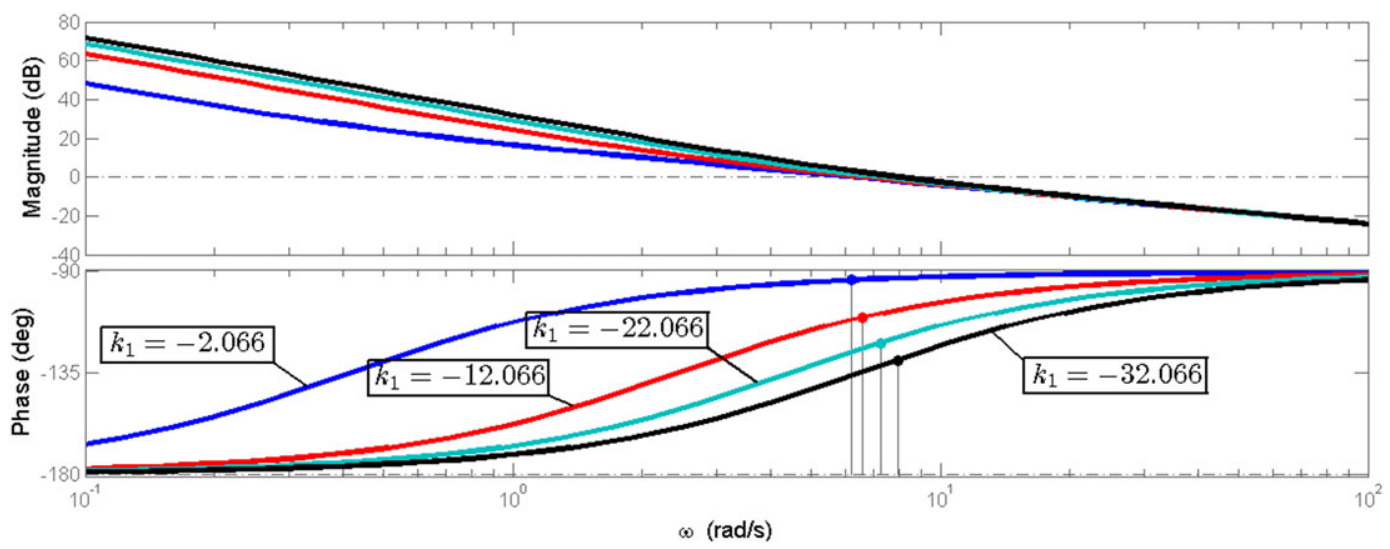

Figure 11. Bode plot for the loop transfer function $L$ with different values of $k_{1}$.

in the presence of bounded disturbance $\left\|d_{L}\right\| \leqslant \delta$.

Let $x_{0} \in \mathbb{R}^{n}$ such that $V_{0} \leqslant 3$ and assume that the system has to meet the performance specification on load disturbances attenuation: $|P S(j \omega)|_{\max } \leqslant 0.8$.

For system (20), the following decomposition is possible:

$$
\begin{aligned}
& P(s)=\frac{1}{s^{2}} \\
& d_{u}(t)=c u^{3}(t) \\
& d=d_{L}(t)+c u^{3}(t) .
\end{aligned}
$$

The results of [9] guarantee stability for the original system (20) in case of stable system (21) and boundedness of input and output signals. Thus, the problem of fulfilling performance specifications for (20) turns to linear problem for stability, disturbance rejection, and ensuring compliance with specifications using results of Section 6.

Choose sampled-time control in the form (5) for $\mu=1$ :

$$
u=k D\left(V_{i}^{-1}\right) x, \quad \text { for } t \in\left[t_{i}, t_{i+1}\right),
$$

where $x=\left[x_{1}, x_{2}\right]^{T}=[y, \dot{y}]^{T}, V_{i}>0: Q\left(V_{i}, x\left(t_{i}\right)\right)=0$. According to Remark 5 of [16], the system (21) is finite-time stable with control scheme (4), (22), and

$$
X H_{\mu}+H_{\mu} X \geqslant \gamma X, \quad \gamma \in \mathbb{R}_{+},
$$

if

$$
|d|=\left|d_{L}+c u^{3}(t)\right| \leqslant \beta \gamma .
$$

At first let us find bound of output signal $x_{1}$. From (3), we can obtain

$$
x D^{2}\left(V^{-1}\right) x \leqslant \frac{1}{\lambda_{\min }(P)} \Rightarrow x_{1}^{2} \leqslant \frac{V^{4}}{\lambda_{\min }(P)}-V^{2} x_{2}^{2} \leqslant \frac{V_{0}^{4}}{\lambda_{\min }(P)} .
$$

Results of the paper [32] allow to find and even select $V_{0}$.

To bound the control signal by $|u| \leqslant u_{0}, u_{0} \in \mathbb{R}_{+}$in addition to (4) and (23), the matrix inequality

$$
\left(\begin{array}{ll}
X & y^{T} \\
y & u_{0}^{2}
\end{array}\right) \leqslant 0
$$

can be added [16]. The inequality (24) is feasible for selected $\beta, \gamma$, and $u_{0}$ satisfying

$$
\delta+|c| u_{0}^{3} \leqslant \beta \gamma .
$$


Thus, solving (4), (23), (25), and (26) and using results presented in previous section, we can consider system (20) to fulfill the frequency specifications based on the Gang of Four.

According to main part of the paper, the easiest way to fulfill the specification $|P S(j \omega)|_{\max } \leqslant 0.8$ is to chose a sufficiently large value of $\left|k_{1}\right|$. However, large value of $\left|k_{1}\right|$ can lead to low-phase margin as in the previous example. Therefore, because

$$
|P S(j \omega)|=\frac{1}{\sqrt{\omega^{4}+\left(2 k_{1}+k_{2}^{2}\right) V^{-2 \mu} \omega^{2}+k_{1}^{2} V^{-4 \mu}}},
$$

then solution of this problem is the lowest value of $\left|k_{1}\right|$ satisfying the system of inequalities

$$
\left\{\begin{array}{l}
k_{1} \leqslant-\frac{0.8^{-2} V_{0}^{4}+0.25 k_{2}^{4}}{k_{2}^{2}} \\
k_{1}<-0.25(1+\mu)(2+\mu)^{2} .
\end{array}\right.
$$

Thus, to satisfy the specification and provide a high-phase margin, the coefficient $k_{1}$ should be chosen equal to -16.3125 . The gain curve of the load disturbance sensitivity function $P S$ for $k_{1}=$ $-16.3125, k_{2}=-3$, and three different values of $V$ is shown in Figure 12. The phase margin is $41^{\circ}$ (Figure 13).

The nonlinear QFT-based method presented in [9] is applicable for similar systems with uncertainties. A disadvantage of this method in comparison with the one presented in this paper is that check of compliance with the established bounds is held posteriori.

It should be noted, that the presented control scheme can also be used in the sense of standard QFT methods. Especially, it may be useful if system has uncertain parameters defined in some ranges. In this case, ILF value $V$ should also be considered as uncertain parameter in the range $V \in\left[V_{\min }, V_{0}\right]$. For instance, the aforementioned example can be solved using QFT method for $c \in\left[a_{\min }, a_{\max }\right]$ and $V \in\left[V_{\min }, V_{0}\right]$.

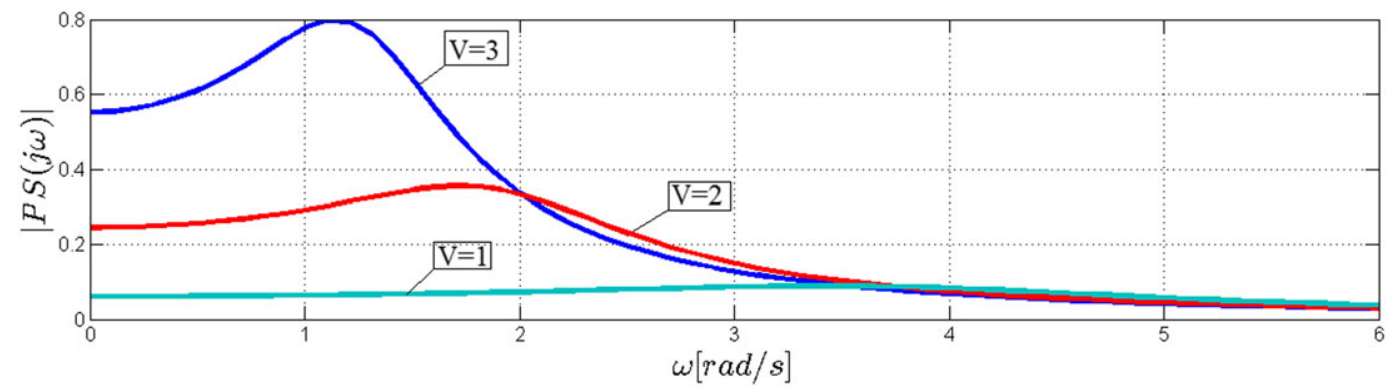

Figure 12. Gain curve of the load disturbance sensitivity function $P S$.

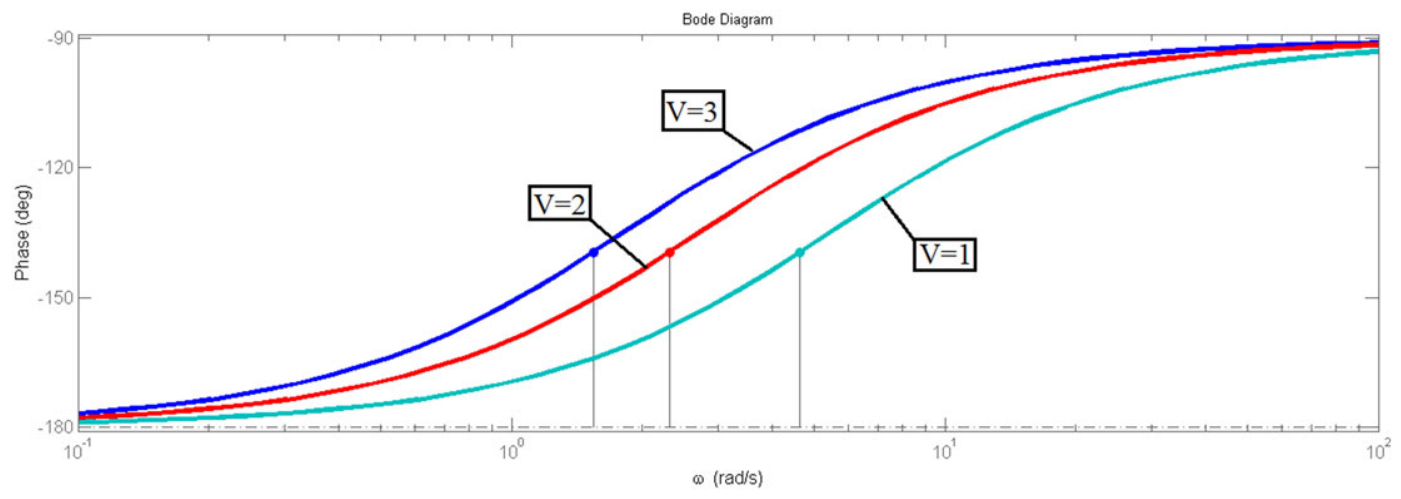

Figure 13. Bode phase plot for the loop transfer function. 


\section{CONCLUSIONS}

The paper presents frequency domain analysis of the finite-time control algorithm presented in [16] in order to make it more attractive for practical implementation. The analysis is performed in order to fulfill different performance specifications based on frequency representation of the Gang of Four and loop transfer function. Applicability of obtained results are supported by examples.

Despite the fact that the control scheme is designed for the multiple integrators system, the frequency domain analysis can be useful in implementation of the control extended to other systems, for example, to MIMO systems as in [19]. Also, it would be of interest to study other specifications, such as specifications based on the Gang of Six, for instance, and to create a special toolbox that can be useful for engineers during designing controllers. These and other aspects are selected as possible directions for further research.

\section{ACKNOWLEDGEMENT}

This study is supported by the Russian Federation Ministry of Education and Science, contract/grant numbers 02.G25.31.0111 and 14.Z50.31.0031.

\section{REFERENCES}

1. Astrom KJ, Murray RM. Frequency Domain Design, Feedback Systems: An Introduction for Scientists and Engineers, Princeton University Press, Princeton, 2008.

2. Fergani N, Charef A. Process step response based fractional $P I^{\lambda} D^{\mu}$ controller parameters tuning for desired closed loop response. International Journal of Systems Science 2016; 47(3):521-532.

3. Horowitz IS. Quantitative Feedback Design Theory: (QFT), Vol. 1. QFT Publication: Boulder, Colorado, 1993: 1-486.

4. Guzman JL, Moreno JC, Berenguel M, Rodriguez F, Sanchez-Hermosilla J. A Frequency domain quantitative technique for robust control system design. In Robust Control, Theory and Applications, Chapter 17. Andrzej Bartoszewicz (Ed.), InTech, Rijeka, Croatia, 2011; 391-405.

5. Chait Y. Proceedings of the 30th IEEE Conference on Decision and Control 1991; 3:2970-2971.

6. Nagamune R. Closed-loop shaping based on Nevanlinna-Pick interpolation with a degree bound. IEEE Transactions on Automatic Control 2004; 49(2):300-305.

7. Nagamune R, Blomqvist A. Sensitivity shaping with degree constraint by nonlinear least-squares optimization. IFAC Proceedings Volumes (IFAC-PapersOnline) 2005; 16:499-504.

8. Baãos A. Nonlinear quantitative feedback theory. International Journal of Robust and Nonlinear Control 2007; 17:181-202.

9. Baãos A, Horowitz IM. Nonlinear quantitative stability. International Journal of Robust and Nonlinear Control 2004; 14:289-306.

10. McFarlane D, Glover K. A loop shaping design procedure using $H_{\infty}$ synthesis. IEEE Transactions on Automatic Control 1992; 37(6):759-769.

11. Levant A. On fixed and finite time stability in sliding mode control. IEEE 52nd Annual Conference on Decision and Control (CDC), Firenze, Italy, 2013; 4260-4265.

12. Cruz-Zavala E, Moreno JA, Fridman L. Fast second-order sliding mode control design based on Lyapunov function. Proceedings of 52nd IEEE Conference on Decision and Control, Firenze, Italy, 2013; 2858-2863.

13. Bernuau E, Perruquetti W, Efimov D, Moulay E. Finite-time output stabilization of the double integrator. Proceedings of 51st IEEE Conference on Decision and Control, Maui, Hawaii, 2012; 5906-5911.

14. Moulay E, Perruquetti W. Finite-time stability and stabilization: state of the art. Lecture Notes in Control and Information Sciences 2006; 334:23-41.

15. Nakamura H. Homogeneous integral finite-time control and its application to robot control. Proceedings of SICE Annual Conference (SICE), Nagoya, Japan, 2013; 1884-1889.

16. Polyakov A, Efimov D, Perruquetti W. Finite-time and fixed-time stabilization: implicit Lyapunov function approach. Automatica 2015; 51:332-340.

17. Chernous'ko FL, Ananevski IM, Reshmin SA. Control of Nonlinear Dynamical Systems: Methods and Applications. Springer-Verlag: Berlin, 2008.

18. Utkin VI, Guldner J, Shi J. Sliding Mode Control in Electro-mechanical Systems (2nd edition). CRC Press: Boca Raton, Florida, 2009: 1-503.

19. Polyakov A, Efimov D, Perruquetti W. Robust stabilization of MIMO systems in finite/fixed time. International Journal of Robust and Nonlinear Control 2016; 26:69-90.

20. Filippov AF. Differential Equations with Discontinuous Right-hand Sides. Kluwer: Dordrecht, 1988.

21. Bhat SP, Bernstein DS. Finite-time stability of continuous autonomous systems. SIAM Journal of Control and Optimization 2000; 38(3):751-766. 
22. Roxin E. On finite stability in control systems. Rendiconti del Circolo Matematico di Palermo 1966; 15(3):273-283.

23. Orlov Y. Finite time stability and robust control synthesis of uncertain switched systems. SIAM Journal of Control and Optimization 2005; 43(4):1253-1271.

24. Zubov VI. Methods of A.M. Lyapunov and Their Applications. Noordhoff Ltd: Groningen, 1964: 1-263.

25. Hermes H. Nilpotent approximations of control systems and distributions. SIAM Journal of Control and Optimization 1986; 24:731-736.

26. Zubov VI. On systems of ordinary differential equations with generalized homogenous right-hand sides. Izvestia vuzov. Mathematica 1958; 1:80-88. (in Russian).

27. Bacciotti A, Rosier L. Lyapunov Functions and Stability in Control Theory, Springer-Verlag: Berlin Heidelberg, 2005: 1-237.

28. Ryan E. Universal stabilization of a class of nonlinear systems with homogeneous vector fields. Systems \& Control Letters 1995; 26:177-184.

29. Bernuau E, Polyakov A, Efimov D, Perruquetti W. Verification of ISS, iISS and IOSS properties applying weighted homogeneity. System and Control Letters 2013; 62(12):1159-1167.

30. Orlov Y. Finite time stability and robust control synthesis of uncertain switched systems. SIAM Journal of Control and Optimization 2004; 43(4):1253-1271.

31. Orlov Y. Discontinuous Systems: Lyapunov Analysis and Robust Synthesis under Uncertainty Conditions. Springer-Verlag: London, 2009.

32. Polyakov A. Time-suboptimal feedback design via linear matrix inequalities. Automation and Remote Control 2015; 76(5):847-862. 